UCRL-JC-130694

PREPRINT

\title{
Emittance Growth in Heavy Ion Rings Due to the Effects of Space Charge and Dispersion
}

\author{
J. J. Barnard, G. D. Craig, A. Friedman, \\ D. P. Grote, B. Losic, S. M. Lund
}

This paper was prepared for submittal to the

Workshop on Space Charge Physics in High Intensity Hadron Rings

Long Island, NY

May 4-7, 1998

June 3, 1998

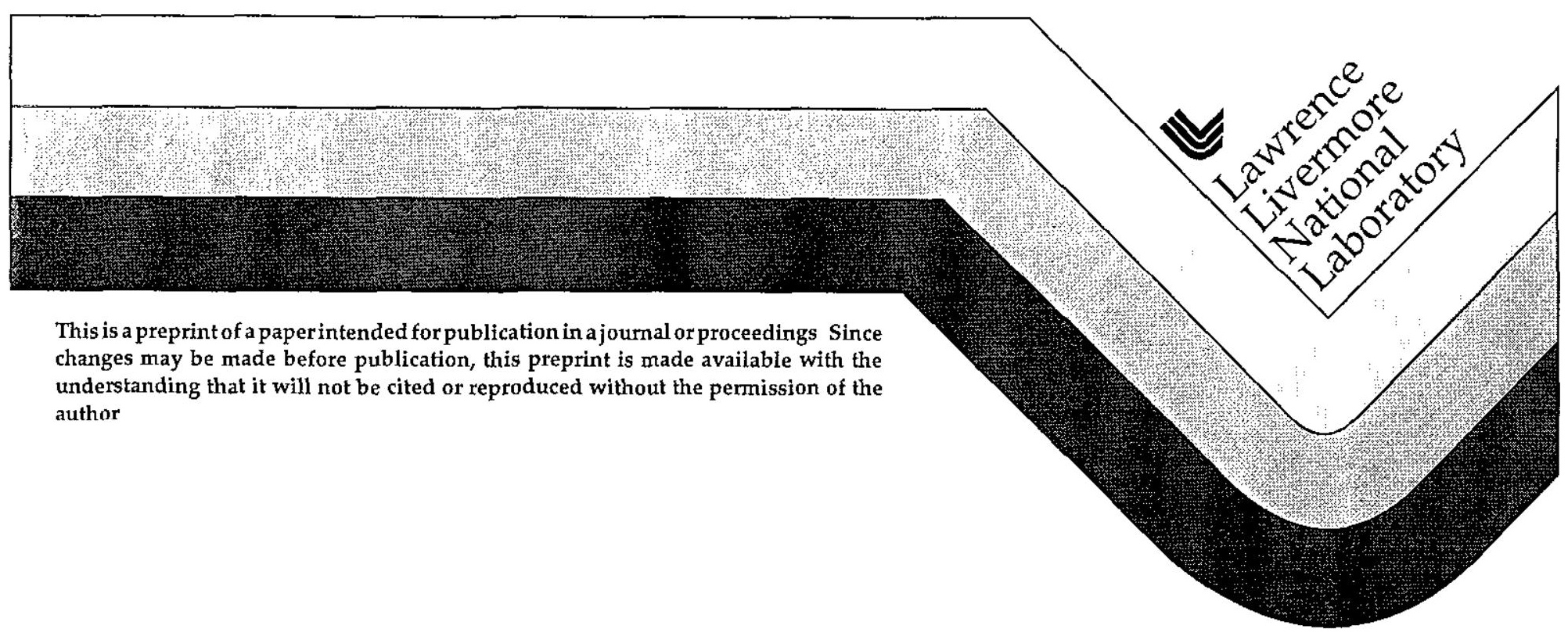




\section{DISCLAIMER}

This document was prepared as an account of work sponsored by an agency of the United States Government. Neither the United States Government nor the University of California nor any of their employees, makes any warranty, express or implied, or assumes any legal liability or responsibility for the accuracy, completeness, or usefulness of any information, apparatus, product, or process

disclosed, or represents that its use would not infringe privately owned rights. Reference herein to any specific commercial product, process, or service by trade name, trademark, manufacturer, or otherwise, does not necessarily constitute or imply its endorsement, recommendation, or favoring by the United States Government or the University of California. The views and opinions of authors expressed herein do not necessarily state or reflect those of the United States Government or the University of California, and shall not be used for advertising or product endorsement purposes. 


\title{
Emittance Growth in Heavy Ion Rings Due to the Effects of Space Charge and Dispersion-
}

\author{
John J. Barnard, George D Craig, Alex Fruedman, \\ David P Grote, Bojan Losic, and Steven M. Lund
}

Lawrence Liver more National Laborator y, L-645, Liver more, CA 94550

\begin{abstract}
We review the derivation of moment equations which include the effects of space charge and dispersion in bends first presented in ref [1] These equations generalize the familiar envelope equations to include the dispersive effects of bends We review the application of these equations to the calculation of the change in emittance resulting from a sharp transition from a straight section to a bend section, using an energy conservation constraint Comparisons of detalled $2 \mathrm{D}$ and $3 \mathrm{D}$ simulations of intense beams in rings using the WARP code (refs $[2,3])$ are made with results obtained from the moment equations We also compare the analysis carried out in ref [1], to more recent analyses, refs $[4,5]$ We further examine self-consistent distributions of beams in bends and discuss the relevance of these distributions to the moment equation formulation
\end{abstract}

\section{Introduction}

There are many applications in which beams having non-negligible space charge forces are transported through bends In heavy ion fusion (HIF), recirculating induction accelerators (recirculators), with large tune depressions, and with rapid acceleration through resonances, are being consıdered to ignite inestial confinement fusion targets Even in linac approaches to HIF, designs of the final transport to the target usually include transport through 180 degrees or more of bend section In some Acclerator Production of Tritium designs, a final bent transport section is being considered as part of an upgrade option For the application of studying high energy density in matter, a beam pulse in a storage ring will be longitudinally compressed, reaching tune shifts for short periods much larg's than allowed by the Laslett-tune shift limit Even in traditional synchrotions and storage rings obeying the Laslett limit, it is useful to have a fiamework in which space charge and dispersion are both inclided

In the HIF application, the not malized emittance of the beam must remain small to be able to focus the beam on a small spot The giowth of the normalized emittance of an accelerated beam is also of interest for many other applications in which high brightness is required The concept of transverse energy conservation was used in ref [1] to study emittance growth in bends This built upon earlier studies which have calculated changes in emittance also using a transverse energy constiaint For example, emittance growth associated witl non-uniform spacecharge distributions was examined in refs [8]- [10] Emittance growth due to mitial beam displacements and mismatches with and without space-charge and momentum spread has been studied in, refs $[11-13,17]$, and references therein

In the work reviewed here the beams propagate in continuous or alternating gradient focusing channel, with phase advances that are depressed due to space

* Work performed under the auspices of the U S Department of Energy by LLNL under contract W-7405-ENG-48 
charge In addition, bends are present, which provide a displacement in the center of oscillation for ions which are off of the design momentum Moment equations are employed to estimate emittance growth arising from the transition from straight sections to bends (See also ref [14] for an estimate of emittance growth due to the transitions in the absence of space charge.) On a transition from a bend to a straight section, or from a straight section to a bend, if the transition is sufficiently sharp, the beam becomes mismatched. We assume that small nonlinear forces act to phase mix particles, and we find the asymptotic emittance of such a beam Further, if we assume that the process of phase mixing is completed before the beam goes through another straight/bend transition, we may calculate the emittance growth through a "racetrack" configuration consisting of two $180^{\circ}$ bends and two stiaight sections, even without a detailed knowledge of the rate at which the phase mixing occurs

\section{Model Equations of Motion}

The force equation in the radial (bend) direction using cylindrical coordinates, $(\rho, \theta, y)$ is

$$
\ddot{\rho}-\frac{v_{\theta}^{2}}{\rho}=F_{b e n d}-v_{\theta}^{2} k_{\beta 0 x}^{2}\left(\rho-\rho_{0}\right)+v_{\theta}^{2} k_{s x}^{2}(\rho-<\rho>)
$$

Here, $\rho$ is the radial coordinate of a particle in a bend, $\theta$ is the azimuthal coordinate, $y$ is the vertical coordinate and $v_{\theta} \equiv \rho \dot{\theta}$ is the azimuthal velocity, and $k_{s x}^{2}$ is a defocusing constant of the assumed linear space charge force in the radial direction (defined below) For simplicity, non-relativistic kinematics are assumed Also, $\rho_{0}$ is the nominal radius of a particle with the azimuthal component of the design momentum $p_{0}$ and design velocity $v_{0} \equiv \beta c$.

The component of the bending force $F_{b e n d}$ in the radial direction is given by

$$
F_{b e n d}=\left(\frac{q e}{A m_{a}}\right) \begin{cases}v_{\theta} B_{y} & \text { (magnetic bends) } \\ E_{\rho} & \text { (electric bends) }\end{cases}
$$

Her $q$ is the ion charge state ( +1 for protons), $e$ is the proton charge in Coulombs, $A$ is the ion mass in amu, $m_{a}$ is the atomic mass unit in $\mathrm{kg} \quad B_{y}$ is the vertical bending field (for magnetic bends) or $E_{\rho}$ is the radial electric field (for electric bends)

We let $x \equiv \rho-\rho_{0}$ and define the increment in path length along the design or bit $d s \equiv \rho_{0} d \theta$ The equations of motion are then given by,

$$
\begin{gathered}
x^{\prime \prime}=-k_{\beta 0 x}^{2}\left(x-x_{m}\right)+k_{s x}^{2}\left(x-x_{c}\right)-\frac{\partial h_{n l}(x, y)}{\partial x} \\
y^{\prime \prime}=-k_{\beta 0 y}^{2} y+k_{s y}^{2}\left(y-y_{c}\right)-\frac{\partial h_{n l}(x, y)}{\partial y} \\
k_{s x}^{2} \equiv \frac{K}{2\left(\Delta x^{2}+\left(\Delta x^{2} \Delta y^{2}\right)^{1 / 2}\right)}, \quad k_{s y}^{2} \equiv \frac{K}{2\left(\Delta y^{2}+\left(\Delta x^{2} \Delta y^{2}\right)^{1 / 2}\right)}
\end{gathered}
$$

Here, $x$ is the in-plane deviation from the design orbit and $y$ is the vertical coordinate in a particular transverse slice of the beam The beam travels in the $+s$ direction, and prime $(')$ indicates derivative with respect to $s, k_{\beta 0 x}$ and $k_{\beta 0 y}$ can represent either alternating gradient focusing (if they are $s$ dependent) or they can represent the focusing effects in the smooth approximation, in which case, $k_{\beta 0 x}=k_{\beta 0 y}=\sigma_{0} / 2 L$ where $\sigma_{0}$ is the undepressed phase advance, and $L$ is the half-lattice period. Dispersion effects enter through the term $x_{m}$, where 
$x_{m} \equiv \cong\left(1 / k_{\beta 0 x}^{2} \rho_{0}\right)\left(\delta p / p_{0}\right)$ for magnetic focusing and $x_{m}=\cong\left(2 / k_{\beta 0 x}^{2} \rho_{0}\right)\left(\delta p / p_{0}\right)$ for electric focusing The quantity $\delta p / p_{0}$ is the fractional difference between the longitudinal momentum of a particle and the design momentum $p_{0}$, and $K \equiv 2 q I /\left(\beta^{3} A I_{o}\right)$ is the perveance Here $I_{o} \equiv 4 \pi \epsilon_{0} m_{a} c^{3} / e$ is the characteristic proton current $(\cong 31$ MA) Finally, for generality, we have included an unspecified external non-linear potential $h_{n l}$ that is a function of $x, y$, and possibly $s$

We adopt the notation of ref [1], throughout this paper in which the quantity $\Delta$ is reserved for the two argument operator in which centroid quantities are subtracted off $\Delta a b \equiv\langle a b\rangle-\langle a\rangle\langle b\rangle$ (e g $\left.\Delta x^{2} \equiv<x^{2}\right\rangle-\langle x\rangle^{2}$ ), where $\left.<\right\rangle$ mincates average over all particles in a slice, $x_{c} \equiv\langle x\rangle$, and $y_{c} \equiv\langle y\rangle$

These equations are identical to the equations found in ref. [1], except here we no longer assume $k_{\beta 0 x}, k_{\beta 0 y}$, and $\rho_{0}$ to be independent of $s$, nor do we require $k_{\beta 0 x}=k_{\beta 0 y}$ In deriving the moment equations in ref [1], no use was made of the assumed constancy or equality of $k_{\beta 0 x}$ and $k_{\beta 0 y}$ nor the constancy of $\rho_{0}$, so the generalization simply amounts to a relabeling of the focusing constants

Eqs (3) and (4) represent, in an approximate way, the effects of linear focusing, linear space charge defocusing, dispersion in a bend, and external nonlinearities in the focusing field. The physical approximations that have been made include the following. (1) Eqs (3) and (4) have been linearized in the small quantities $k_{\beta 0} x, k_{\beta 0} y$, and $\delta p / p_{0}$ (The non-linear term $h_{n l}$ has also been included in some of the derivations) (2) The non-linearity is small $\left(\left|h_{n l}\right|<<\left|k_{\beta 0 x}^{2} x^{2}\right|,\left|k_{\beta 0 y}^{2} y^{2}\right|\right)$ (Terms which are non-linear in $\delta p / p_{0}$, such as $k_{\beta_{0}} x \delta p / p_{0}$, have been neglected.) (3) Space charge forces depend only on lowest order moments (We have used the KV formula for the electrostatic potential, which is equivalent to assuming uniform density elliptical beam Centroid position and semi-major axes are, however, allowed to vary with $s$ ). (4) The beam is coasting $\left(p_{0}, \beta\right.$, and $\delta p$ are constants) (5) The beam is non-relativistic $(\beta<<1)$

Let $f\left(x, x^{\prime}, y, y^{\prime}, \frac{\delta p}{p_{0}}, s\right)=\mathrm{d} N / \mathrm{d} x \mathrm{~d} x^{\prime} \mathrm{d} y \mathrm{~d} y^{\prime} \mathrm{d} \frac{\delta p}{p_{0}}$ where $\mathrm{d} N$ is the number of particles within incremental phase volume $\mathrm{d} x \mathrm{~d} x^{\prime} \mathrm{d} y \mathrm{~d} y^{\prime} \mathrm{d} \frac{\delta_{p}}{p_{0}} \quad$ For the model equations (3)-(5) the Vlasov equation becomes.

$\frac{\partial f}{\partial s}+x^{\prime} \frac{\partial f}{\partial x}+\left(-k_{\beta 0 x}^{2}\left(x-x_{m}\right)+k_{s x}^{2}\left(x-x_{c}\right)-\frac{\partial h_{n l}}{\partial x}\right) \frac{\partial f}{\partial x^{\prime}}+y^{\prime} \frac{\partial f}{\partial y}+\left(-k_{\beta 0 y}^{2} y+k_{s y}^{2}\left(y-y_{c}\right)-\frac{\partial h_{n l}}{\partial y}\right) \frac{\partial f}{\partial y^{\prime}}=0$

The average of a variable $\xi$ over the contumous distribution is given by

$$
<\xi>(s) \equiv \int \mathrm{d} x \int \mathrm{d} x^{\prime} \int \mathrm{d} y \int \mathrm{d} y^{\prime} \int \mathrm{d} \frac{\delta p}{p_{0}} \xi f\left(x, x^{\prime}, y, y^{\prime}, \frac{\delta p}{p_{0}}, s\right) / N,
$$

where

$$
N \equiv \int \mathrm{d} x \int \mathrm{d} x^{\prime} \int \mathrm{d} y \int \mathrm{d} y^{\prime} \int \mathrm{d} \frac{\delta p}{p_{0}} f\left(x, x^{\prime}, y, y^{\prime}, \frac{\delta p}{p_{0}}, s\right)
$$

Following ref [7], we take all second order moments of the Vlasov eq (6), yielding eight (first-order with respect to $s$ ) coupled moment equations

$$
\begin{aligned}
& \frac{\mathrm{d}}{\mathrm{d} s} \Delta x^{2}=2 \Delta x x^{\prime} \\
& \frac{\mathrm{d}}{\mathrm{d} s} \Delta x^{\prime 2}=\left(-2 k_{\beta 0 x}^{2}+2 k_{s x}^{2}\right) \Delta x x^{\prime}+2 k_{\beta 0 x}^{2} \Delta x^{\prime} x_{m}-2 \Delta\left(x^{\prime} \frac{\partial h_{n l}}{\partial x}\right) \\
& \frac{\mathrm{d}}{\mathrm{d} s} \Delta x x^{\prime}=\Delta x^{\prime 2}-k_{\beta 0 x}^{2} \Delta x^{2}+k_{s x}^{2} \Delta x^{2}+k_{\beta 0 x}^{2} \Delta x x_{m}-\Delta\left(x \frac{\partial h_{n l}}{\partial x}\right) \\
& \frac{\mathrm{d}}{\mathrm{d} s} \Delta y^{2}=2 \Delta y y^{\prime} \\
& \frac{\mathrm{d}}{\mathrm{d} s} \Delta y^{\prime 2}=\left(-2 k_{\beta 0 y}^{2}+2 k_{s y}^{2}\right) \Delta y y^{\prime}-2 \Delta\left(y^{\prime} \frac{\partial h_{n l}}{\partial y}\right) \\
& \frac{\mathrm{d}}{\mathrm{d} s} \Delta y y^{\prime}=\Delta y^{2}-k_{\beta 0 y}^{2} \Delta y^{2}+k_{s y}^{2} \Delta y^{2}-\Delta\left(y \frac{\partial h_{n l}}{\partial y}\right) \\
& \frac{\mathrm{d}}{\mathrm{d} s} \Delta x x_{m}=\Delta x^{\prime} x_{m} \\
& \frac{\mathrm{d}}{\mathrm{d} s} \Delta x^{\prime} x_{m}=-k_{\beta 0 x}^{2} \Delta x x_{m}+k_{s x}^{2} \Delta x x_{m}+k_{\beta 0 x}^{2} \Delta x_{m}^{2}-\Delta\left(x_{m} \frac{\partial h_{n l}}{\partial x}\right)
\end{aligned}
$$


Similarly, the first order moments of eq (6) yield the following

$$
\begin{aligned}
& \frac{\mathrm{d}}{\mathrm{d} s} x_{c}=x_{c}^{\prime} \\
& \frac{\mathrm{d}}{\mathrm{d} s} x_{c}^{\prime}=-k_{\beta 0 x}^{2} x_{c}+k_{\beta 0 x}^{2}<x_{m}>-<\frac{\partial h_{n L}}{\partial x}> \\
& \frac{\mathrm{d}}{\mathrm{d} s} y_{c}=y_{c}^{\prime} \\
& \frac{\mathrm{d}}{\mathrm{d} s} y_{c}^{\prime}=-k_{\beta 0 y}^{2} y_{c}-<\frac{\partial h_{n L}}{\partial y}>
\end{aligned}
$$

Note that if $h_{n i}=0$, eq (7) forms a closed sets of 8 equations, and eq (8) forms two sets of two closed equations If $h_{n l} \neq 0$, eqs (7) and (8) form the beginning of an infinite hierarchy of moment equations

\section{Transverse Energy Conservation}

For the case of alternating gradient focusing, and when the bends occupy only a fraction of the lattice, the focusing constants $k_{\beta 0 x}^{2}, k_{\beta 0 y}^{2}$, and the bend radius of curvature $\rho_{0}$ are dependent on $s$ This $s$-dependence of the external forces implies that there will not be a constant energy-like quantity However, as in ref [1], if $k_{\beta 0 x}, k_{\beta 0 y}$, and $\rho_{0}$ are constants representing average quantities, we may define a transverse energy $H$ :

$$
\begin{gathered}
2 H=k_{\beta 0 x}^{2} \Delta x^{2}+k_{\beta 0 y}^{2} \Delta y^{2}+\Delta x^{2}+\Delta y^{2}-2 k_{\beta 0 x}^{2} \Delta x x_{m}-K \ln \left(\left(\Delta x^{2}\right)^{1 / 2}+\left(\Delta y^{2}\right)^{1 / 2}\right) \\
+2<h_{n l}>+k_{\beta 0 x}^{2} x_{c}^{2}+k_{\beta 0 y}^{2} y_{c}^{2}+x_{c}^{\prime 2}+y_{c}^{\prime 2}
\end{gathered}
$$

Use of eqs (7) and (8) shows that

$$
\frac{\mathrm{d}}{\mathrm{d} s} H=\frac{\mathrm{d}}{\mathrm{d} s}<h_{n l}>
$$

Thus if $h_{n l}$ is not a function of $s, H$ is an invariant

\section{Emittance Growth}

We define separate $x$ and $y$ emittances

$$
\epsilon_{x}^{2} \equiv 16\left(\Delta x^{2} \Delta x^{\prime 2}-\Delta x x^{\prime 2}\right), \quad \epsilon_{y}^{2} \equiv 16\left(\Delta y^{2} \Delta y^{\prime 2}-\Delta y y^{\prime 2}\right)
$$

Uising eqs (7), the following emittance evolution equations can be derived

$$
\begin{gathered}
\frac{\mathrm{d}}{\mathrm{d} s} \epsilon_{x}^{2}=32 k_{\beta 0 x}^{2}\left(\Delta x^{2} \Delta x^{\prime} x_{m}-\Delta x x^{\prime} \Delta x x_{m}\right)+32\left(\Delta\left(x \frac{\partial h_{n l}}{\partial x}\right) \Delta x x^{\prime}-\Delta x^{2} \Delta\left(x^{\prime} \frac{\partial h_{n l}}{\partial x}\right)\right) \\
\frac{\mathrm{d}}{\mathrm{d} s} \epsilon_{y}^{2}=32\left(\Delta\left(y \frac{\partial h_{n l}}{\partial y}\right) \Delta y y^{\prime}-\Delta y^{2} \Delta\left(y^{\prime} \frac{\partial h_{n l}}{\partial y}\right)\right)
\end{gathered}
$$

Thus the emittance would be constant if non-linearties were not present $\left(h_{n l}=0\right)$ and the momentum spread were absent $\left(x_{m}=0\right.$ for all particles ) Eqs (12) and (13) are valid for both continuous and alternating gradient focusing 


\section{Equilibrium Beam}

In the continuous focusing approximation, the left-hand-sides of eqs (7) and (8) can be set to zero to obtain the following equilibrium (constant moment) conditions

$$
\begin{aligned}
& \Delta x^{\prime 2}=\left(k_{\beta 0 x}^{2}-k_{s x}^{2}\right) \Delta x^{2}-k_{\beta 0 x}^{2} \Delta x x_{m}+\Delta\left(x \frac{\partial h_{n t}}{\partial x}\right) \\
& \Delta y^{\prime 2}=\left(k_{\beta 0 y}^{2}-k_{s y}^{2}\right) \Delta y^{2}+\Delta\left(y \frac{\partial h_{n l}}{\partial y}\right) \\
& \Delta x x_{m}=\frac{k_{\beta 0 x}^{2} \Delta x_{m}^{2}-\Delta\left(x_{m} \frac{\theta h_{n x}}{\partial x}\right)}{k_{\beta 0}^{2}-k_{x x}^{2}} \\
& x_{c}=<x_{m}>-\frac{1}{k_{\beta 0 x}^{2}}<\frac{\partial h_{n x}}{\partial x}> \\
& y_{c}=-\frac{1}{k_{\beta 0 y}^{2}}<\frac{\partial h_{n l}}{\partial y}> \\
& \Delta x x^{\prime}=\Delta y y^{\prime}=\Delta x^{\prime} x_{m}=\Delta\left(x^{\prime} \frac{\partial h_{n l}}{\partial x}\right)=\Delta\left(y^{\prime} \frac{\partial h_{n l}}{\partial y}\right)=0 \\
& x_{c}^{\prime}=y_{c}^{\prime}=0
\end{aligned}
$$

Assuming that $h_{n l}=x_{c}=y_{c}=0$ the transverse energy (9) in equilibrium $\left(H=H_{e q}\right)$ reduces to

$$
2 H_{e q}=\left(2 k_{\beta 0 x}^{2}-k_{s x}^{2}\right) \Delta x^{2}+\left(2 k_{\beta 0 y}^{2}-k_{s y}^{2}\right) \Delta y^{2}-\frac{3 k_{\beta x 0}^{4} \Delta x_{m}^{2}}{k_{\beta 0 x}^{2}-k_{s x}^{2}}-K \ln \left(\left(\Delta x^{2}\right)^{1 / 2}+\left(\Delta y^{2}\right)^{1 / 2}\right)
$$

Note that for a given $H_{e q}$, the ratio of $\Delta x^{2}$ to $\Delta y^{2}$ is still unspecified A further assumption is required to specify the final state of the beam. It is often reasonable to assume that transverse energy equipartition results in a beam in which the two transverse temperatures are equal, i.e. $\Delta x^{2}=\Delta y^{\prime 2}$. (Note that we have implicitly assumed that the timescale for complete equipartition $\left[\Delta x^{\prime 2}=\Delta y^{\prime 2}=\Delta\left(\delta p / p_{0}\right)^{2}\right]$ is much larger than timescales of interest) The condition that $\Delta x^{\prime 2}=\Delta y^{\prime 2}$ can be expressed as a relation between $\Delta y^{2}$ and $\Delta x^{2}$.

$$
\Delta y^{2}=\frac{\left(k_{\beta 0 x}^{2}-k_{s x}^{2}\right)}{\left(k_{\beta 0 y}^{2}-k_{s y}^{2}\right)} \Delta x^{2}-\frac{k_{\beta 0 x}^{4}}{\left(k_{\beta 0 x}^{2}-k_{s x}^{2}\right)} \Delta x_{m}^{2}
$$

When $k_{\beta 00 x}=k_{\beta 0 y}=k_{\beta 0}$ and $\Delta x_{m}^{2}<<x^{2}$ this result reduces to.

$$
\Delta y^{2} \cong \Delta x^{2}-2 k_{\beta 0}^{4} \Delta x_{m}^{2} /\left(k^{2}\left(k^{2}+k_{\beta 0}^{2}\right)\right) \text {, }
$$

where $k^{2} \equiv k_{\beta 0}^{2}-K /\left(4 \Delta x^{2}\right)$

\section{Rings}

Suppose a beam is in equilibrium on a stranght section, and then enters a continuous bend ( $\mathrm{e}$ e a ring) If the lattice parameters (such as the bend 1 adius of curvature) abruptly change to new values, the beam becomes mismatched to the bend Physically, particles that are not on the design momentum for the bend initially become spatrally separated, creating non-linear space-charge forces, allowing phase mixing of the coherent mismatch oscillations until a new equilibium is reached For concreteness, we consider a lattice in which $k_{\beta 0 x}=$ $k_{\beta 0 y} \equiv k_{\beta 0}$, and also assume that $k_{\beta 0}$ is the same both in the straight section and in the bend section Thus, in this example, we assume the contribution to focusing from the bends is included in $k_{\beta 0}$ We assume that the initial beam (subscript 0) is matched to the straight section Thus $\Delta y_{0}^{2}=\Delta x_{0}^{2}$, and $\Delta x_{0}^{\prime 2}=\Delta y_{0}^{\prime 2}$ $=k_{0}^{2} \Delta x_{0}^{2}$, and all other moments are equal to zero The initial transverse energy satisfies $2 H_{0}=\left(2 k_{\beta 0}^{2}+2 k^{2}\right) \Delta x_{0}^{2}-K \ln \left[2\left(\Delta x_{0}^{2}\right)^{1 / 2}\right]$ To calculate the final equilibrium beam parameters, we set the final transverse energy equal to the initial transverse 
energy, and smultaneously solve this constrant with the equalized temper ature constrant, eq (16) Thus, there are two equations in two unknowns $\left(\Delta x^{2}\right.$ and $\left.\Delta y^{2}\right)$, yielding the final equilibrum values of $\Delta x^{2}$ and $\Delta y^{2}$ in the bend From the equilibrium values (eq 14), all other second order moments may be calculated, mcluding the emittance

In ref [3], comparisons of the results of the continuous focusing theory were made to 2D WARP PIC simulations of the transition from straight to bend for parameters of a small recirculator experiment being built at Lawrence Livermore National Laboratory The relevant parameters of the simulation were the ion species was singly charged Potassium, (mass 39), at an energy of $80 \mathrm{kV}$, and a current of $2 \mathrm{~mA}$, leading to a perveance $K$ of $354 \times 10^{-4}$ The average focusing constants are $k_{\beta 0 x}=k_{\beta 0 y}=189 \mathrm{~m}^{-1}$, corresponding to a phase advance of 78 degrees and half-lattice perıod $L=036 \mathrm{~m}$. The average bend radius of curvature 1s $\rho_{0}=229 \mathrm{~m}$. The normalized emittances $\epsilon_{n x} \equiv \gamma \beta \epsilon_{x}$ and $\epsilon_{n y}=\gamma \beta \epsilon_{y}$ (where $\gamma$ is the Lor ent $z$ factor of the beam) were both set to $003 \mathrm{~mm}$-mrad at injection into the $\operatorname{lng} . \delta p / p_{0 r m s}=72 \times 10^{-4}$ was assumed

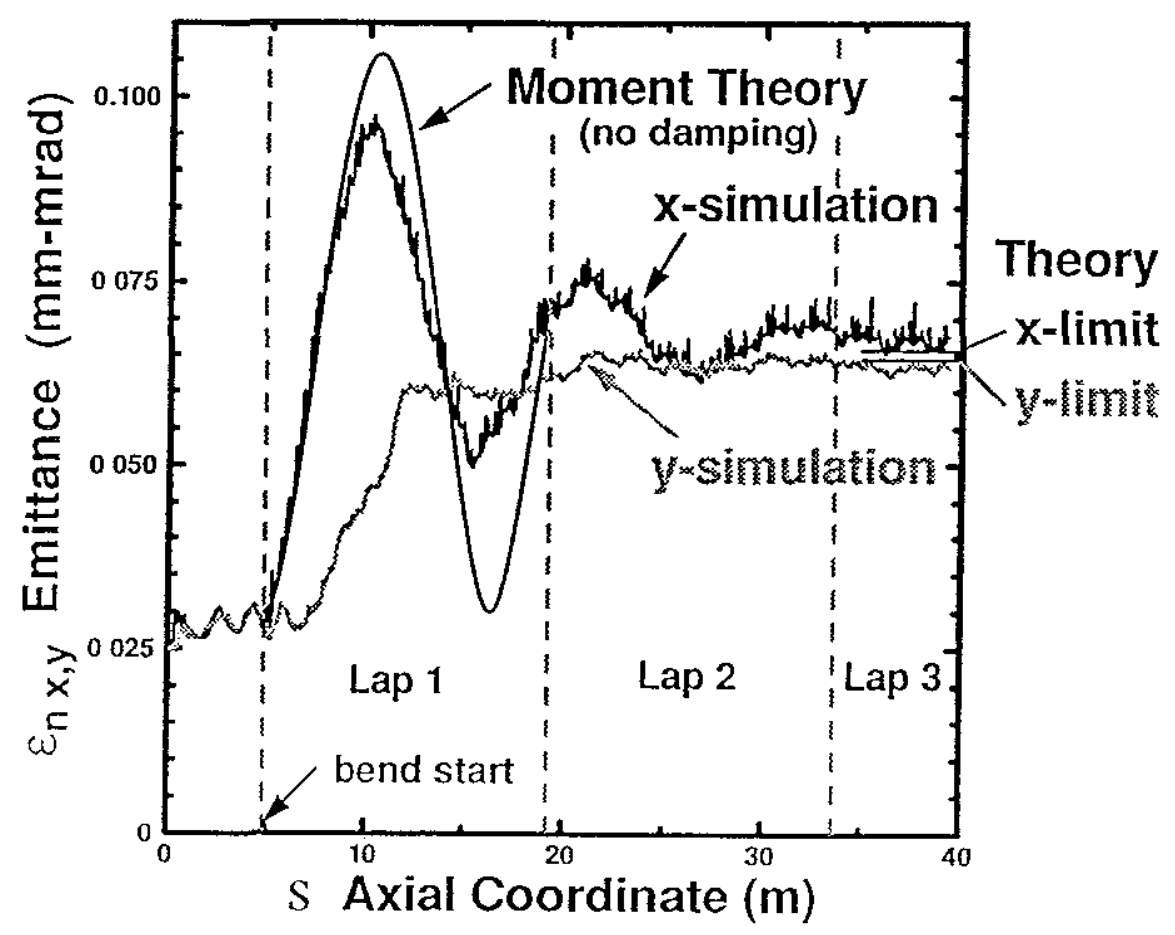

Figure 1. Comparison of WARP simulations with model results (from ref [3]) $x$ and $y$ emittance evolution in a ing geometry, after mitialization in a straight section, as calculated by WARP, by direct integration of the moment equations (Moment Theory, no damping) and the asymptotic value using energy conservation

In Figure 1, (from ief [3]), we plot the WARP simulations of the normalized 2 and $y$ emittances over 3 laps of the small recirculator. In addition, we plot the initial evolution of the emittance as predicted by direct integration of the moment equations, (indicated as Moment Theory (no damping)) in the figure The simulations include all of the details of alternating gradient lattice including fringe fields and image effects, as well as the non-linear space charge fields The 
theory calculations use only the unifom focusing and bending approxumation Also, because the moinent equations do not include non-linearities and the associated non-linear phase mixing, the amplitude of the $x$-emittance oscillations remain constant and the $y$-emittance does not grow In the simulations, small non-linearities cause the oscillations to damp and the $y$-emittance to gradually grow closer to the $x$-emittance Although direct integration of the moment equations does not capture the damping of the oscillations in the $x$-emittance or the growth in the $y$-emittance, the moment equations accurately predict the initial amplitude and frequency of the oscillations Also shown on the figure are the final equilibrum results (indicated as $\mathrm{x}$-limit theory, and $\mathrm{y}$-limit theory) calculated using the prescription indicated above, which is based on using the moment equations to calculate the transverse energy and assuming equality of the final velocity spreads in the $x$ and $y$ drections As can be seen, the theory closely predicts the asymptotic values of the $x$ and $y$ emittances as found by the fully 3D simulations, and also captures the simulation result that the $y$-emittance equilibrates to a value less than the $x$-emittance Simulations of the University of Maryland Ring (ref [15]) shows a similar increase in emittance with an ultimate saturation.

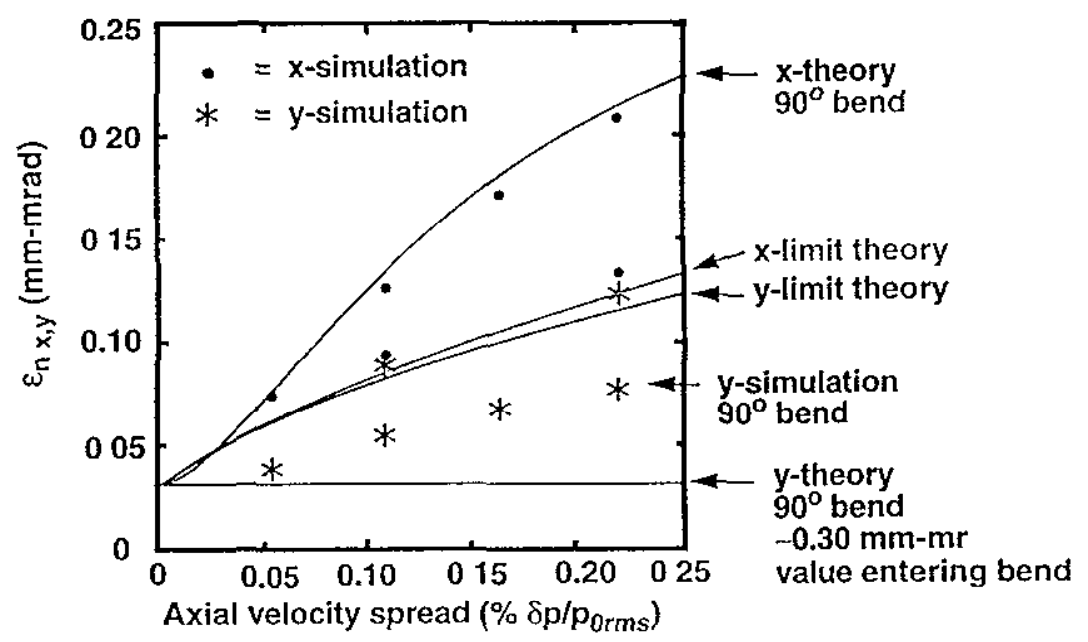

Figure 2. Comparıson of WVARP smulations with model results (fion y ef [3]) $x$ and $y$ einittance after the 90 regi ee bend and asymptotic results as a function of the fractional momentum spread (in percentage), after intialization in a stiaight section, as calculated by WARP, by direct integiation of the moment equations ( $x$ - and $y$ - theor y $90^{\circ}$ bend) and the asymptotic value using energy conser vation $(x-$ and $y$ - limit theory)

In Figure 2, (from ref [3]), we plot the emittance at the end of nunety degiees of bend and also the asymptotic values of the emittance, all as a functron of $\left(\Delta\left(\delta p / p_{0}\right)^{2}\right)^{1 / 2} \equiv \delta p / p_{0 r m s}$ This quantity is largely unknown in the experiment, and it could range anywhere from the value expected from accelerative cooling (see e $g$ ref [16]) of the longitudinal momentum spread induced by the $\sim 01 \mathrm{eV}$ ion source, which results in a spread of orde1 $10^{-6}$, or if the fractional error in the injector diode voltage er rors is as large as 0005 (at high enough frequency), the resulting induced fractional momentum spread, $\delta p / p_{0 r m s}$ would be as large as $25 \times 10^{-3}$ A third possible source of momentum spread comes from instabilities associated 
with an ansotropic velocity distribution (ref [22]) If this instability heats the longitudinal component until it is of the same temperature as the transverse, the resulting moment spread would be $\delta p / p_{0 r m s} \cong \epsilon_{x} / 4\left(\Delta x^{2}\right)^{1 / 2} \cong 72 \times 10^{-4}$ (For a more complete discussion of Ief [3]) As can be seen from the plot, direct integration of the moment equations closely captures the simulation value of the emittance after 90 degrees (during the initial emittance oscillation) and closely matches the mean ruse in emittance and difference between the $x$ and $y$ emittances

When $k_{\beta 0 x}=k_{\beta 0 y}$, and when the change in emittance is much less than the original emittance, one may analytically estimate the change in emittance squared in an abrupt transition from straight to bend

$$
\epsilon_{x}^{2}-\epsilon_{x 0}^{2} \cong 16 \frac{k_{\beta 0}^{4}\left(2 k_{\beta 0}^{2}+k^{2}\right)}{k^{2}\left(k_{\beta 0}^{2}+k^{2}\right)} \Delta x_{0}^{2} \Delta x_{m}^{2}, \quad \epsilon_{y}^{2}-\epsilon_{y 0}^{2} \cong 16 \frac{k_{\beta 0}^{6}}{k^{2}\left(k_{\beta 0}^{2}+k^{2}\right)} \Delta x_{0}^{2} \Delta x_{m}^{2}
$$

Eqs (18) are valid only for small changes in emittance, and so are not applicable to the parameters of figure 1

\section{Racetracks}

In a recirculator that is composed of two $180^{\circ}$ bends connected by two straight sections in the shape of a racetrack, if phase mixing is rapid enough the equilibrium can be reached before each transition Transverse energy is conserved as a beam enters a bend from a straight, but since the beam acquires a finite $\Delta x x_{m}$ as it finds equilibrium in the bend, the transverse energy will be discontinuous entering a straight from a bend, (where $\rho_{0}$ becomes infinite, and hence $\Delta x x_{m}$ abruptly changes to zero ) The quantities $\Delta x^{2}, \Delta x^{\prime 2}, \Delta y^{2}, \Delta y^{\prime 2}$ are, of course, continuous at all transitions A new value of $H$ is calculated which is again constant throughout the straight section At the beginning of the bend the process repeats In Fig (3), we have applied this formulation to a small scale racetrack recirculator, which is not undergoing acceleration This prescription for calculation of the emittance was carried out numerically, and compared with the $3-\mathrm{D}$ version of the WARP code As can be seen, the emittance growth is tracked closely although the higher frequency oscillatory behavior associated with lattice and mismatch oscillations are, of course, not seen (For small values of $\Delta\left(\delta p / p_{0}\right)^{2}$, or large values of $\sigma / \sigma_{0}$ the prescription over estimates the emittance growth, since the assumption of complete phase-mixing between transitions is not acheved )

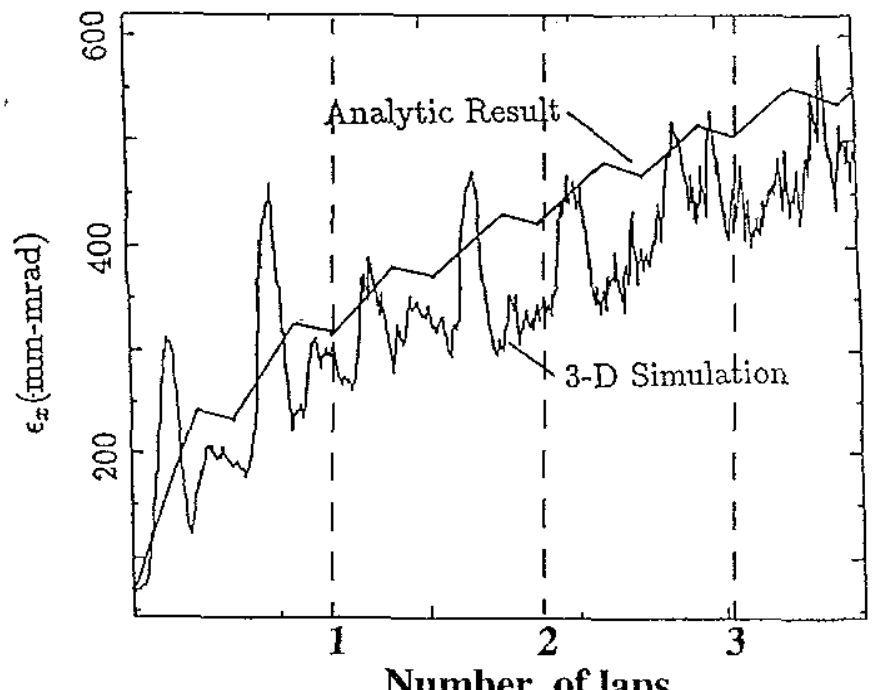

Figure 3. Emittance growth in a racetrack. The parameters are $\sigma_{0}=72^{\circ}, \sigma=8^{\circ}$, 
$\rho=36 \mathrm{~m}, A m_{a} \beta^{2} c^{2} / 2=10 \mathrm{MeV},\left(\Delta\left(\delta p / p_{0}\right)^{2}\right)^{1 / 2}=00079$ The length of each straight section was $72 \mathrm{~m}$

\section{Comparison to Other Recent Work}

Recently, in ref $[4,18]$, envelope equations which include the same physics (i e dispersion linear in $\delta p / p_{0}$, and space charge with an assumed elliptical symmetry) as the moment equations (eqs. 7,8) were derived Venturini and Reiser found a generalized emittance $\epsilon_{d x}$ which, when expressed in terms of the notation of this paper can be written as

$$
\epsilon_{d x}^{2}=\left(\Delta x_{m}^{2} \Delta x^{2}-\Delta x x_{m}^{2}\right)\left(\Delta x_{m}^{2} \Delta x^{2}-\Delta x^{\prime} x_{m}^{2}\right)-\left(\Delta x_{m}^{2} \Delta x x^{\prime}-\Delta x x_{m} \Delta x^{\prime} x_{m}\right)^{2}
$$

By taking the derivative of $\epsilon_{d x}^{2}$ and using eqs (7), it is straightforward to show that $\epsilon_{d x}^{2}$ is constant. By using the constancy of $\epsilon_{d x}$ and $\epsilon_{y}$, two of the eight first order moment equations could be eliminated, leaving six first order moment equations, equivalent to the three second order equations of ref [4] Thus the envelope equations in ref [4] contain the same physical content as the previously derived (ref. [1]) moment equations. In ref. [4], it is suggested that it would be possible to eliminate much of the growth in emittance by matching the envelope and the dispersion function $D(s)=\left(\Delta x \delta p / p_{0}\right) / \Delta\left(\delta p / p_{0}\right)^{2}$ (as defined in ref. [4]). This is equivalent to finding the matched periodic solution of the moment equations in the ring, and then constructing the section which injects the beam into the ring such that the values of the moments match those of the matched periodic solution within the ring In order to prevent mismatch oscillations of the centroid, the centroid equations (8) must also be matched on the transition from straight to bend Another method (ref [1]) of preventing emittance growth is by slowly varying the radius of curvature, allowing an adiabatic transition into the bend.

Also, recently in ref [5], vertical and horizontal dispersion functions are derived The horizontal dispersion function derived by Lee is identical to that of ref [4], except that horizontal/vertical coupling is allowed such as can occur if there are quadrupole rotation errors (see e $\mathrm{g}$ ref [19]) The vertical dispersion function is identical to that of a straight lattice, but again with the inclusion of horizontal/vertical coupling The envelope equations derived are not consistent with the moment eqs $(7,8)$ or the envelope equations of ref [4], however, due to additional approximations

\section{Self-consistent distributions}

Recently, in ref [20], a self-consistent $\mathrm{KV}$ solution to the Vlassov-Poisson system in a bend was obtained. The solutions in the non-relativistic case are of the form

$$
f\left(x, x^{\prime}, y, y^{\prime}, \delta p / p_{0}\right)=f_{\perp}\left(H_{1}\right) \exp \left[-\left(\delta p / p_{0}\right)^{2} / \delta_{0}^{2}\right]
$$

Here $2 H_{\perp}=x^{\prime 2}+y^{\prime 2}+k_{\beta 0}^{2} x^{2}+k_{\beta 0}^{2} y^{2}+2 q \phi / m v_{0}^{2}-2\left(x / \rho_{0}\right) \delta p / p_{0}$ In ref. [20], generalizations to the $\mathrm{KV}$ distribution were investigated of the form $f_{\perp}\left(H_{\perp}\right)=f_{0} \delta\left(H_{\perp}-H_{0}\right)$ In ref [21] thermal equilibrium distributions of the form $f_{\perp}\left(H_{\perp}\right)=f_{0} \exp \left(-H_{\perp} / k_{b} T\right)$ have been examined. Figure 4 illustrates the two distributions for the parameters of the University of Maryland electron ring experiment (ref. [20]) $\left(k_{\beta 0}^{2}=17437\right.$ $\mathrm{m}^{-2}$, current $=105 \mathrm{~mA}$, energy $=10 \mathrm{keV}, \rho_{0}=182 \mathrm{~m}$, with $k / k_{\beta 0}=033$, and $\left.\delta p / p_{0 r m s}=01\right)$

The moment eqs require averages over $x E_{x}$ and $y E_{y}$ where $E_{x}$ and $E_{y}$ are the electric field components due to space charge Although in ref [1], an ellipse with uniform charge density was used to calculate $E_{x}$ and $E_{y}$, as pointed out in ref [7], the results also apply if the density is a function only of $x^{2} / \Delta x^{2}+$ 
$y^{2} / \Delta y^{2}$, i e constant on nested elliptical surfaces (ref [4]) As can be seen for the generalized KV distribution, the assumption of a density distribution that is constant on nested ellipses is poor for the KV distribution, but appears to be a better approximation for thermal equilibrium beams, which underlies the calculation of asymptotic emittance growth above This may, in part, explain why the WARP simulation results agree well with the moment model.

\section{a). KV distribution}
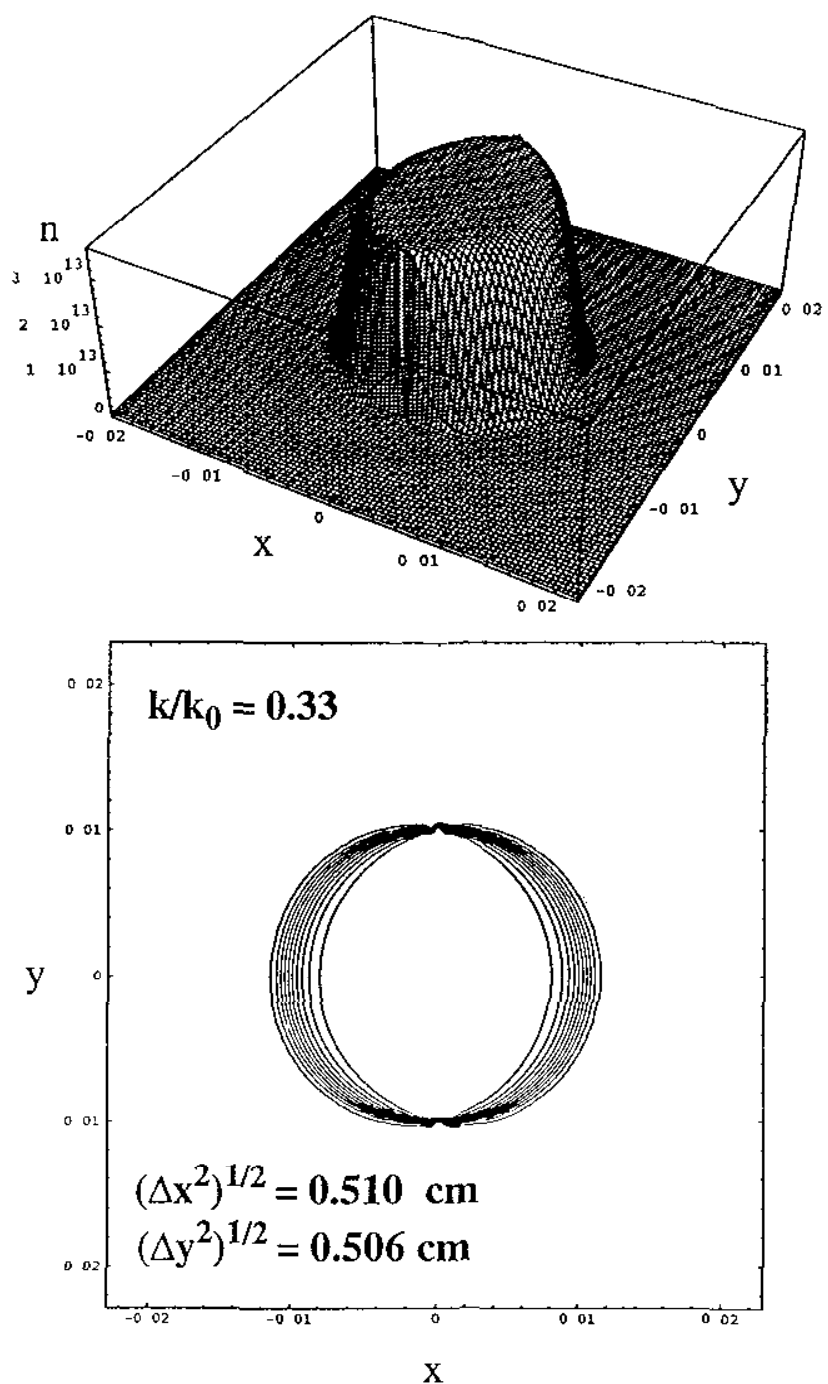

\section{b). Thermal equilibrium distribution}
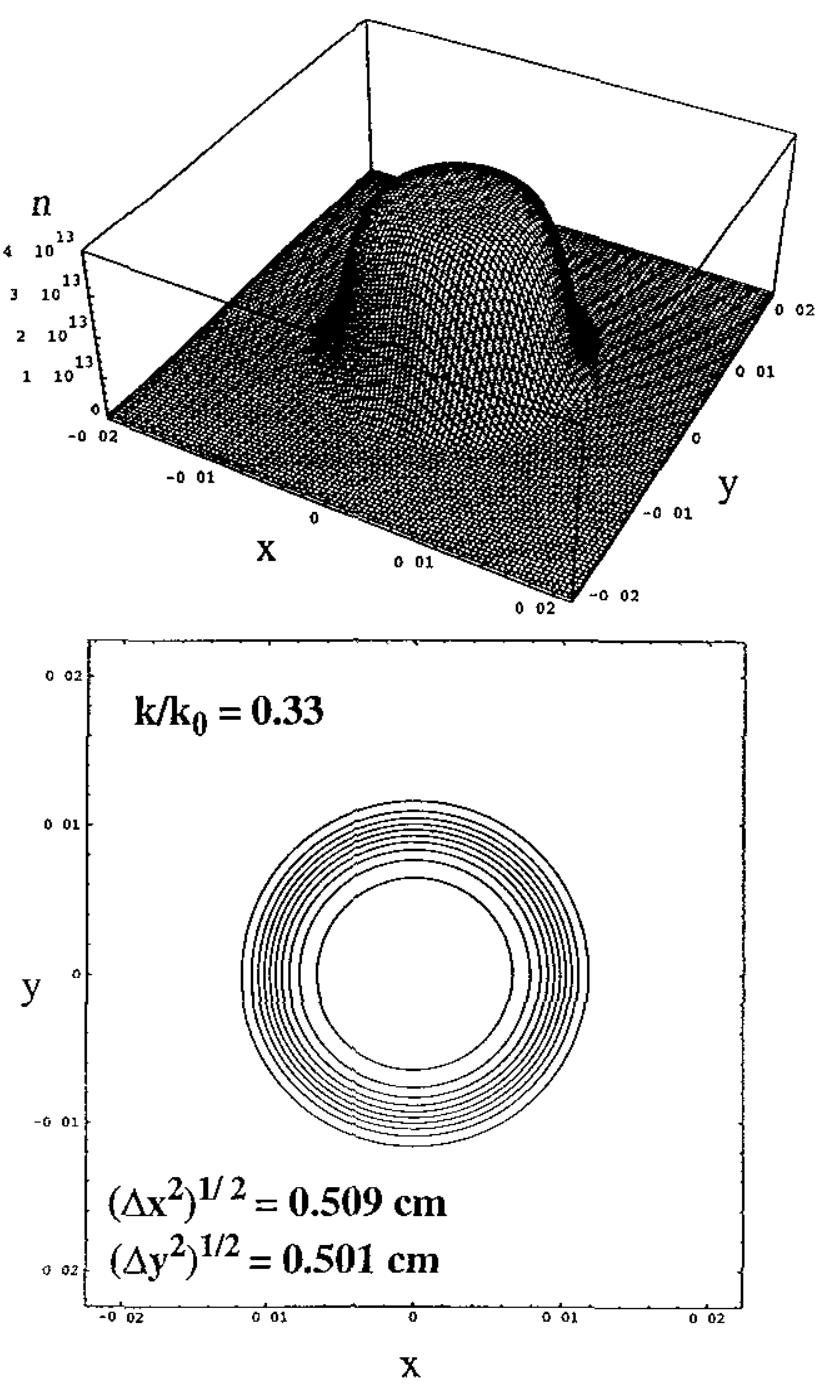

Figure 4. Self consistent beam density distributions in bends a) Surface plot (upper) and contour plot (lower) of generalized KV distribution (ref [20]), and b) Thermal distribution (ref [21])

\section{Discussion}

Emittance growth from sharp transitions, as discussed above, provides one source of emittance growth Others, such as misalignments of quadrupoles, field strength errors, non-linear applied fields, etc, provide additional mechanisms to degrade the emittance In the recirculator design of ref [6], an insertion and extraction region occurs over a $100 \mathrm{~m}$ long stralght section, which gives the 
machine some of the features of the racetrack in that equilibration can occur on passing from bend to straight and from straight to bend Since the energy is increasing on each lap it would be difficult to design the insertion/ extraction section which is "matched" at all energies Assuming abrupt transitions, use of the moment equations together with the parameters of the beam at the exit of the High Energy Ring of ref [6] lead to an estimated emittance growth by a factor of about 2 Since the entrance beam parameters lead to a much smaller emittance growth, the normalized emittance will grow by less than a factor of 2 This is within the emittance "budget" in the design of ref [6] It is also possible that the transitions between bends and straights can be made gradual enough so that equilibria are reached adiabatically, with little associated growth in the noi malized enittance

\section{Conclusions}

We have reviewed the derivation of moment equations in which focusing, space charge, and dispersion in a bend, are included We have shown that the moment equations derived in ref [1], using the average bend and continuous focusing approximation, accurately predicts the initial amplitude and frequency of emittance oscillations which occur at a sharp transition from a straight section to a bend (ref [3]) We have also reviewed the method of estimating the asymptotic value of the emittance growth due to straight/bend mismatches from considerations of transverse energy conservation as the beam equilibrates. By assuming the transverse energy of the beam is conserved during the equilibration, and assuming that the beam reaches equilibrium, and also that the equilibrium transverse velocity spread is the same in $x$ as it is in $y$ we can calculate all moments and thus the change in emittance In racetracks, in which fou such transitions are made per lap, we have calculated the emittance growth under the assumption that the equilibrium state is reached between each transition In small scale rings the analytic result agreed well with $2-\mathrm{D}$ and 3-D WARP simulations when $\sigma / \sigma_{0}$ was small and the velocity spread was sufficiently large (so that the assumption of phase mixing between transitions was realized). In the High Energy Ring of ref [6], this prescription yielded an emittance growth of less than a factor of 2

\section{References}

1 J J Barnard, H D Shay, S S. Yu, A Frıedman, and D P Grote, "Emittance Growth in Heavy-Ion Recirculators,"1992 Lmear Acceler ator Conference Proreedings 1992 Angust 24-28, Ottawa, Ontarı, Canada, C R Hoffman, ed (1992) AECL 10728 (AECL Research, Chalk River, Canada) p 229

2 A Filedman, D P Grote, D A Callahan, A B Langdon, I Haber, "3D Particle Simulations of Axially Confined Heavy Ion Beams U'sung the WARP code Transport Around Bends," Particle Accelerators, 37, 131, (1992)

3 S M Lund, J J Barnard, G D Craig, A Friedman, D P Grote, H S Hopkins, T C. Sangster, W M Sharp, S Eylon, T J Fessenden, E Henestroza, S Yu, and I Haber, "Numetical Simulation of Intense-Beam Experiments at LLNL" and LBNL," Nuclear Instruments and Methods A (in press)

4 Masco Venturini and Martin Reıser, "RMS Envelope Equations in the Presence of Space Charge and Dispersion," submitted to Phys Rev Letters (1998)

5 S Y Lee and H Okamoto, "Space Charge Dominated Beams in Synchrotrons," these proceedings (1998)

6 J J Barnaid, F Deadrick, A Friedman, D P Grote, L V Griffith, H C Kirbie, V K Nell, M A Newton, A C Paul, W M Sharp, H D Shay, R O Bangerter, A Faltens, C G Fong, D L Judd, E P Lee, L L Reginato, S S 
Yu, and T F Godlove, "Recirculating Induction Accelerators as Drivers for Heavy Ion Fusion," Physics of Fluids B Plasma Physics, 5, 2698 (1993) Also, "Study of Recirculating Induction Accelerators as Drivers for Heavy Ion Fusion," Lawrence Liver more National Laboratory UCRL-LR-108095 (1992)

7 F J Sacherer, "RMS Envelope Equations with Space Charge," IEEE Transactions on Nuclear Science NS-18, 1105, (1971)

8 P. M Lapostolle, "Possible Emittance Increase through Filamentation Due to Space Charge in Continuous Beams," IEEE Transactions on Nuclear Science, NS-18, 1101, (1971)

9 T P Wangler, $\mathrm{K}$ R Crandall, $\mathrm{R}$ S Mills, and M Reiser, "Relation Between Field Energy and RMS Emittance in Intense Particle Beams," IEEE Transactions on Nuclear Science, NS-32, (1985)

10 O. A Ander son, "Internal Dynamics and Emittance Growth in Space-ChargeDominated Beams," Particle Acclerators, 21, 197, (1987)

11 J J. Barnard, "Anharmonic Betatron Motion in Free Electron Lasers" Nuclear Instruments and Methods in Physics Research A296 (1990)

12 M Reiser "Free Energy and Emittance Growth in Nonstationary Charged Partıcle Beams," Journal of Applied Physics 70, 1919 (1991).

13 O A Anderson, "Emittance Growth Rates for Displaced or Mismatched High Current Beams in Nonlinear Channels," Proc of the Fourth NPB Techn Symp , Argonne National Laboratory, (1992)

$14 \mathrm{~K}$ T Nguyen "Emittance Growth and Energy Bandwidth in the IFRR," Proceedings of the 1990 DARPA/ SDIO/Services Annual Charged Part Beam Review, p 71, Nav Res Lab., Washington D C (1991)

15 R A Kishek, I Haber, M Venturini, and M Reiser, "PIC Code Simulations of the Space-Charge-Dominated Beam in the Unversity of Maryland Election Ring," these proceedings

16 M Reiser, "Theory and Design of Charged Particle Beams," (Wiley \& Sons, New York, 1994)

17 J J Barnard, J. Miller, I Haber, "Emittance Growth in Displaced Space Charge Dominated Beams with Energy Spread," Proceedings of the 1993 Particle Accelerator Conference, Washington, D C, May 1993, 5, 3612 (1993)

18 M Venturini, R A Kishek, and M Reiser, "Dispersion and Space Charge," these proceedings (1998)

19 John J Barnard "Emitance Growth from Rotated Quadrupoles in Heavy Ion Accelerators," Proceednngs of the 1995 Particle Accelerator Confer mnce, Dallas TX, held May 1-5, 1995 (5), 3241 (1996)

$20 \mathrm{M}$ Venturm and M Reiser, "Self-consistent beam distributions with space charge and dispersion in a curcular ring lattice," Phys Rev E, 574725. (1998)

21 John J Barnard, and Bojan Losic, "Emittance Growth from Bend/Straight transitions for Beams approaching Thermal Equilibinum," Proceedings of the 1998 Linear Acceler ator Conference," In Preparation (1998)

22 I Haber, D A Callahan, A Finedman, D P Grote, S M Lund, T F Wang, "Characteristics of an Electrostatic. Instability Driven by TransverseLongitudnal Temperatu1c Anısotropy", Nuclear Instruments and Methods $A$, in press (1998) 


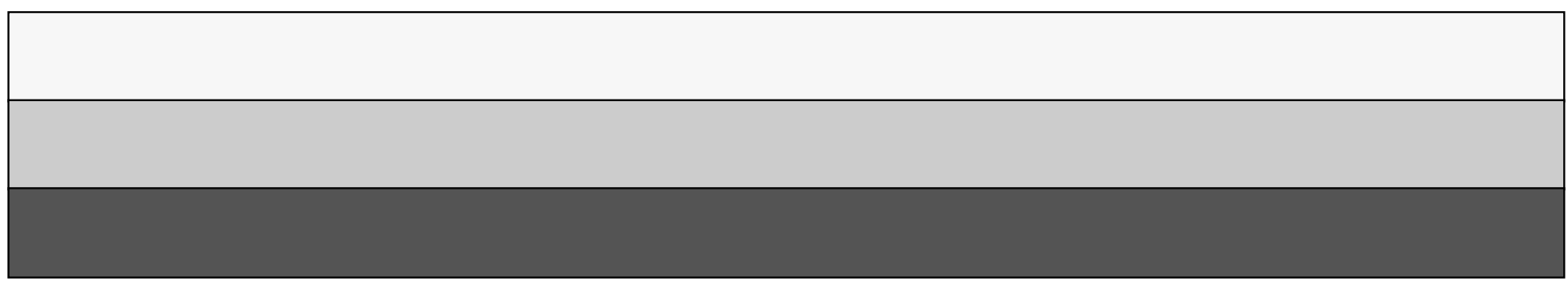

